\title{
A Rare Case of Ovarian Apocrine Adenocarcinoma
}

\author{
Liping $\mathrm{Xu}^{1}$, Zhi $\mathrm{Ma}^{1}$, Keya Wanga ${ }^{1}$ and Ying Yang*2 \\ ${ }^{1}$ Medical College of Qingdao University, People's Republic of China \\ ${ }^{2}$ Department of Gynecology, Yantai Yuhuangding Hospital affiliated to Qingdao University, Republic of China
}

Received: May 11, 2018; Published: May 22, 2018

*Corresponding author: Ying Yang, Department of Gynecology, Yantai Yuhuangding Hospital, No.20 East Yuhuangding Road, Yantai 264000, Shandong, China

\begin{abstract}
Mature cystic teratoma of the ovary is the most commen benign ovrian tumor. Malignant mature teratomas(MCT) is rare, occurring in only $1 \%$ to $2 \%$ of cases, the main transfer pathway is the spread and direct infiltration of the abdomen, and Lymph node metastasis is rare. So far, there only 2 case of apocrine adenocarcinoma arising from a mature teratoma has been reported in the English literature. We report the first known case of an apocrine adenocarcinoma arising within an ovarian mature cystic teratoma which with pelvic lymph node metastasis. The patient recurrent during postoperative chemotherapy, similar to malignant teratoma in malignancy, different from MCT but similar to cutaneous apocrine carcinoma and the apocine carcinoma of breast in the way of transfer.
\end{abstract}

\section{Case Study}

A 46-year-old woman was admitted to the emergency department on Nov. 14th 2015 with the complaint of "palpable right lower abdomen mass with abdominal pain for 3 days". Her past menstrual periods were regular, LMP: 2015-10-12, gestational history: 2-0-3-2, the last parturition was 13 years ago, with subsequent IUD contraception. Pelvic examination: a $10 * 8$ * $8 \mathrm{~cm}$ cystic mass could be palpated at the left side of the adnexal area, with clear border, poor activity and tenderness; no obvious abnormality was found at the right side, but with tenderness and rebound tenderness. Pelvic MRI (3T) scanning: a giant solid-cystic mass was found to the upper left side of the uterus, it was polycystic with clear edge and nonuniform internal signal, the largest diameter was about $12 * 11 \mathrm{~cm}$; pelvic fluid was detected; thus, malignant tumor deriving from the left adnexa with pelvic fluid was diagnosed. Laboratory examinations: TSGF: 66.4 U / ml, CEA: 6.07U / ml, CA125: 59.02U / ml, CA15-3: 49.03U / ml, CA19-9: 7.02U / ml, CYFRA: 48.42U / ml, NSE: 21.56U / ml, CA72-4:> 300U / ml. According to the pelvic and auxiliary examinations, it's more likely to be malignant ovarian carcinoma, so we carried out "laparotomy".

Intraoperative findings: a giant pelvic cyst originating from the left ovary was found, irregular shape, like three kinds of tumors' fusion; one part of it was like hemorrhagic cysts, about $5 \mathrm{~cm}$ in diameter, the right side was teratoma with a diameter of about $4 \mathrm{~cm}$. Bottom of the cyst was a cauliflower-like mass, about $4 \mathrm{~cm}$ in diameter. The overall mass was solid- cystic with broken surface surrounded by old clot. The tumor adhered firmly to pelvic peritoneum, uterine wall and rectum; The uterus was in a normal size, but several hard nodules could be palpated from the postuterine wall to Douglas pouch, ranging about $4 \mathrm{~cm}$; right ovary increased, its size was about $5 * 4 * 4 \mathrm{~cm}$ with irregular shape, smooth surface and intact wall; metastatic nodular lesions could be found at both paracolic sulci and part of the mesenteric surface. Postoperative pathology: cancer tissue could be found within left adnexa and marked pelvic lesions. Left adnexa: malignant transformation of ovarian teratoma, presenting apocrine carcinoma-like differentiation. Right adnexa: infiltration of apocrine carcinoma, mainly because of the metastasis from left side. Cancer metastasis could be found within 1/11 marked left pelvic lymph nodes; no metastasis was found within 5 marked lymph nodes at the right side and the omentum majus. Immunohistochemical results: GCDFP-15 (+), AR (+), P53 (+), Ki-67 was about 50\%, CK7 $(+)$, CK20 (-), Villin (-), GHTA- ), PAX-8 (-), CDX2 (-), ER (-), P63 (-), CK5 / $6(-)$, P16 (-), her2 (-) (Figure 1), supporting the diagnosis of ovarian apocrine carcinoma. Postoperative diagnosis: malignant transformation of ovarian teratoma-apocrine carcinoma IIIc stage.

\section{Treatment}

Postoperative reexamination of tumor test series: TSGF:75.9U/ $\mathrm{ml}$, CEA:2.22U/ml,CA125: 14.49U/ml,CA15-3 : 15.50U/ml, CA19-9 : 7.61U/ml, CYFRA:16.44U/ml, NSE:21.05U/ml, CA72-4 : $21.93 \mathrm{U} / \mathrm{ml}$. The postoperative TP regimen (paclitaxel liposomes 
$300 \mathrm{mg}+$ nedaplatin $120 \mathrm{mg}$, intravenous chemotherapy) was applied for 6 cycles, before the 6th cycle, auxiliary examination results showed: CA72-4: 22.49U / ML, CA125: 9.46U / ML, enhanced CT scanning for the pelvic and abdominal cavity: a block of slightly lower-density shadows with ambiguous border could be found within caudete lobe of the liver, lesions were unevenly enhanced with radiocontrast, the largest one was $2.6 * 3.7 \mathrm{~cm}$ (Figure 2a); small cystic low-density shadow with clear border was found in the upper pole of the spleen, lesions could be slightly enhanced with radiocontrast; a small increased- density nodular shadow with clear border was detected within uterine area, its diameter was about $2.1 \mathrm{~cm}$ and could be enhanced with radiocontrast; multiple lymph nodes were found in retroperitoneal and pelvic cavity, the largest one was about $1.1 \mathrm{~cm}$ in diameter. Thus indicated the recurrence of the tumor, since there was no enhanced CT or MRI scanning for the liver (before operation) and pelvic cavity (after operation), it's difficult to evaluate the chemotherapy by simple tumor-marker examination without imaging information; the patient completed the sixth cycle of TP regimen.

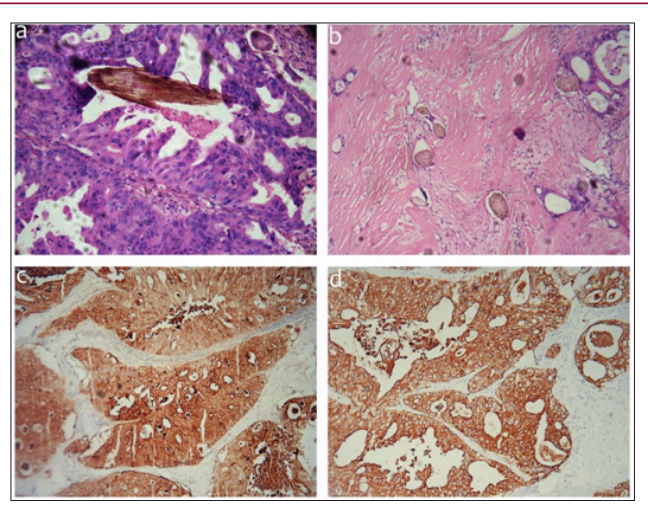

Figure 1: visible hair cuticle, may originated from teratoma; no other teratoma components, perhaps already phagocytized by cancer cells; vacuolar nuclei, prominent nucleoli, granular and eosinophiliccytoplasm (Figure 1a, 1b). Immunohistochemical stain for GCDFP-15 (Figure 1c) and CK7 (Figure 1-d) showing strong diffuse staining of tumor cells.

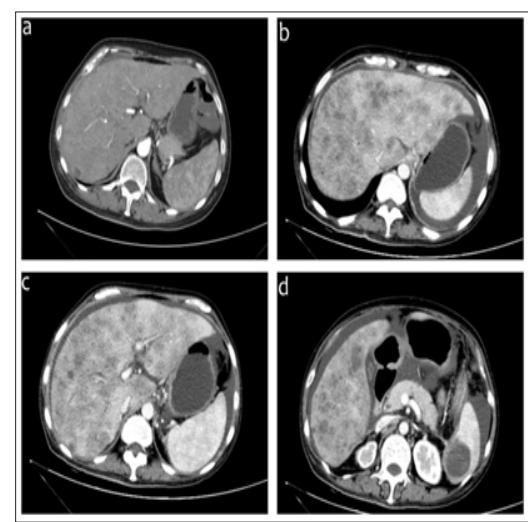

Figure 2: CT scanning first revealed recurrence of the tumor and metastasis (about $38.37^{*} 26.85 \mathrm{~mm}$ ) to the liver (Figure 2-a); before third-line chemotherapy, CT scanning showed multiple quasi-circular low-density shadows within the liver and spleen, most metastases could be slightly enhanced with radiocontrast.
This patient was transferred to Fudan University Shanghai Cancer Center because of the progress of the disease, she was advised to recieve PC regimen (Paclitaxel-albumin 200mg d1, $100 \mathrm{mg} \mathrm{d} 8+$ carboplatin 500mg d2). Laboratory examinations after 2 cycles of chemotherapy: blood: WBC: $2.19 * 109$ / L, neutrophils: $1.06 * 109$ / L, GGT:469U/L, AST:83 U/L, ALT:52 U/L , indicating II degree of myelosuppression and III degree damage of the liver function. After the application of Ruibai(G-CSF) and other liverprotection medications, myelosuppression was significantly improved while liver function was still abnormal that possibly caused by carboplatin, then a single paclitaxel liposome (albuminbinding paclitaxel $100 \mathrm{mgd} 1, \mathrm{~d} 8$ ) was chose for the subsequent chemotherapy. After 3 cycles, liver function recovered a lot than before, 1 cycle of PC regimen (Paclitaxel-albumin $200 \mathrm{mg}$ d1, d8 + carboplatin $400 \mathrm{mg} \mathrm{d} 2$ ). Reexamination of enhanced CT scanning for the abdominal and pelvic cavity: the liver is larger with scattered slightly lower-density nodular shadows inside, this nodules were in different size and with unclear borders, margins were mildly enhanced with radiocontrast; a quasi-circular low-density shadow was found within spleen, about $3.7 \mathrm{~cm}$ in diameter and the margin could be slightly enhanced with radiocontrast; multiple irregular soft-tissue-density shadows were found in retroperitoneal, abdominal and pelvic cavity, they had unclear borders and could be unevenly enhanced with radiocontrast, perihepatic and pelvic fluid was also detected; line-like shadows with high density was found in rectal area see. Tumor-markers: CA15-3: 25.60U / ML, CEA: 5.87ng / ML, CA125: 100.1U / ML, CA72-4: 255.3U / ML. Considering the chemotherapy resistance, second-line protocol (bevacizumab 400mg ivgtt + gemcitabine $1.4 \mathrm{~g} \mathrm{~d} 1$, d8) was chose, but chemotherapy side effects like diarrhea, abdominal pain and hypoproteinemia appeared after 2 cycles. These adverse effects were eventually relieved after symptomatic and supportive treatments' application. Enhanced CT scanning for peritoneal cavity: metastatic tumors in the liver were more and larger than before, slightly increased in the spleen; intra-abdominal metastases had no significant changes than before while there was increased ascites (Figure 2b-2d). Tumor-markers: CA15-3: 22.22U / ML, CEA: 5.12ng / ML, CA125: 172.2U / ML, CA72-4: 230.5U / ML prompted the further development of this disease, and then the secondline chemotherapy was replaced with docetaxel $60 \mathrm{mg} \mathrm{d} 1, \mathrm{~d} 8+$ Lobaplatin $30 \mathrm{mg} \mathrm{d} 2$. The disease was currently progressing rapidly because of the chemotherapy resistance, and there have been multiple metastases of pelvic organs and lymph nodes, especially the diffuse lesions within liver and spleen. ECOG score was 3 points.

\section{Discussion}

Squamous cell carcinoma accounts for the most part of malignant transformation of ovarian mature cystic teratoma, followed by adenocarcinoma, and apocrine carcinoma is an extremely rare type of adenocarcinoma. To the best of our knowledge, there are only 2 case reports about ovarian apocrine carcinoma worldwide. In 1987, W Yasui et al. [1] reported the first case of sweat gland carcinoma originating from ovarian teratoma, but it turned out to be eccrine not apocrine adenocarcinoma. Actually, it's Morimitsu Y6 who reported the first case of apocrine adenocarcinoma of the ovary 
in 1993; more recently, Melissa Holmeset al. [2] reported another case of ovarian apocrine adenocarcinoma with supraclavicular lymph node metastasis, while there was no detailed information in their report about pelvic and abdominal metastases, and more importantly, the specific treatment and prognosis. There are very limited reports about the malignant transformation, recurrence and metastasis of ovarian mature cystic teratoma, it's generally believed to be mainly confined within the pelvic cavity, blood and distant metastasis is rare. So whether the distant metastasis is a feature of ovarian apocrine adenocarcinoma is still uncertain.

Since the degree of malignant transformation of ovarian mature teratoma is normally high, there are no unified standards of postoperative adjuvant therapy for it yet. So far, operation is still the first choice as no other therapeutic protocol has been proved to be more effective. Given the special metastasis of this tumor, which can infiltrate directly and implant on peritoneum, paraaortic lymph nodes and pelvic metastases should be carefully and thoroughly resected after radical hystero-oophorectomy, postoperative radiotherapy or chemotherapy can be applied according to patients' condition. Palenzuela et al. [3] argued that comprehensive staging for operation assisted with postoperative chemotherapy can bring more benefits for patients with Malignant Ovarian Germ Cell Tumor (MOGCT). For the treatment of patients with stage above Ic, some scholars believe that chemotherapy for malignant germ cell tumors should not be used as adjuvant therapy but select different regimens depends on the origin of the tumor 2. Unfortunately, there are no specific chemotherapy regimens for apocrine adenocarcinoma yet.

Literatures about apocrine adenocarcinoma of the breast are relatively rich; there is no much difference of the treatments between apocrine adenocarcinoma and many other types of breast cancer: surgery, chemotherapy, radiotherapy and endocrine therapy. According to the literature, breast apocrine adenocarcinoma and invasive ductal carcinoma have similar prognosis [4,5]. Generally, sweat adenocarcinoma of the skin is invasive, the most common metastasis is via local lymph nodes or a distant metastasis [6-8]. Studies have shown that the metastasis and recurrence of dermal sweat adenocarcinoma have some correlation with the malignancy of primary tumors $[4,5]$. It's almost certain that there is a higher positive rate of lymph nodes metastases for both dermal sweat and breast apocrine adenocarcinoma [4,6-8] but whether lymph node metastasis and (or) a distant metastasis is the significant feature of apocrine adenocarcinoma deriving from mature terotoma is still unclear as a lack of literature resources [9-10].

Postoperative diagnosis of the patient admitted to our hospital: ovarian apocrine adenocarcinoma deriving from mature cystic teratoma, stage IIIc. Due to involvement of the right ureter, ureteral anastomosis and Double-J stent placement were performed during the operation. Repeated urine occult blood made it not suitable for radiotherapy. PEB (cisplatin, etoposide and bleomycin) regimen is appropriate for ovarian germline tumor, but this patient presented characteristics of epithelial tumor for lymph nodes metastases were found in intra- and postoperative pathology. Then chemotherapy regimen which usually for ovarian epithelial tumor was applied as the supplementary treatment, while tumors still tended to be recurrent, refractory and resistant to chemotherapy. Progression of the disease was finally out of control despite five chemotherapy regimens were used successively. At present, enhanced CT and MRI scanning of the pelvic as well as abdominal cavity showed multiple organs, lymph nodes and peritoneal metastases, no metastasis to the lung was identified with enhanced CT scanning of thoracic cavity. Metastases to distant organs and lymph nodes were not clear for the patient's refusal to PET-CT examination.

Besides, there is no postoperative enhanced CT or MRI examination of the pelvic and abdominal cavity, evaluation of the chemotherapy can not be made based on pre- and postoperative imaging information even with tumor-markers examination which is not so reliable. So whether the current recurrence of such ovarian apocrine adenocarcinoma was from the incomplete resection during operation, or the tumor was insensitive to chemotherapy is still uncertain. Poor prognosis of this patient may be associated with the following factors:

a) Ovary is a pelvic organ comparing to the breast, direct invasion and implantation of the tumor are more likely to happen, surgical removal of the tumor requires higher technical skills;

b) It had already developed into IIIc stage at the time of surgery, multiple organs had been involved;

c) There is no uniform regimen for apocrine adenocarcinoma yet, we used chemotherapy normally for ovarian epithelial tumor in this case, which turned out to be poorly targeted. According to the postoperative pathological findings, ovarian apocrine adenocarcinoma of this case was well-differentiated, which is similar to highly differentiated breast apocrine adenocarcinoma. Perhaps we can seek better regimens from chemotherapy protocols for breast cancer.

\section{References}

1. W Yasui, T Kameda, D Harada, H Nakatani, JHata, et al. (1987) a Case of Sweat Gland Carcinoma Arising in a Mature Cystic Teratoma of the Ovary. Gan No Rinsho 33: 875-880.

2. M Holmes, T Robb (2017) A Rare Case of Primary Apocrine Adenocarcinoma Arising within a Mature Cystic Teratoma of the Ovary, Metastatic to a Supraclavicular Lymph Node. Int J Gynecol Pathol.

3. G Palenzuela, E Martin, A Meunier, P Beuzeboc, V Laurence, et al. (2008) Comprehensive Staging Allows for Excellent Outcome in Patients with Localized Malignant Germ Cell Tumor of the Ovary. Ann Surg 248: 836841.

4. MA den Bakker, AC Ansink, PC Ewing-Graham (2006) Cutaneous-Type Angiosarcoma Arising in a Mature Cystic Teratoma of the Ovary. J Clin Pathol 59: 658-660.

5. KL Hollowell, SC Agle, EE Zervos, TL Fitzgerald (2012) Cutaneous Apocrine Adenocarcinoma: Defining Epidemiology, Outcomes, and Optimal Therapy for a Rare Neoplasm. J Surg Oncol 105: 415-419.

6. CJ Ko, AJ Cochran, W Eng, SW Binder (2006) Hidradenocarcinoma: A Histological and Immunohistochemical Study. J Cutan Pathol 33: 726730 .

7. Y Morimitsu, O Nakashima, Y Nakashima, M Kojiro, T Shimokobe (1993) Apocrine Adenocarcinoma Arising in Cystic Teratoma of the Ovary. Arch Pathol Lab Med 117: 647-649. 
8. H Takeuchi, K Tsuji, H Ueo, T Kano, Y Maehara (2004) Clinicopathological Feature and Long-Term Prognosis of Apocrine Carcinoma of the Breast in Japanese Women. Breast Cancer Res Treat 88: 49-54.

9. E Belin, K Ezzedine, S Stanislas, N Lalanne, M Beylot Barry, et al. (2011) Factors in the Surgical Management of Primary Eccrine Porocarcinoma:
Prognostic Histological Factors Can Guide the Surgical Procedure. Br J Dermatol 165: 985-989.

10. K Tanaka, S Imoto, N Wada, N Sakemura, K Hasebe (2008) Invasive Apocrine Carcinoma of the Breast: Clinicopathologic Features of 57 Patients. Breast J 14: 164-168.
(C) 7 This work is licensed under Creative

Submission Link: https://biomedres.us/submit-manuscript.php

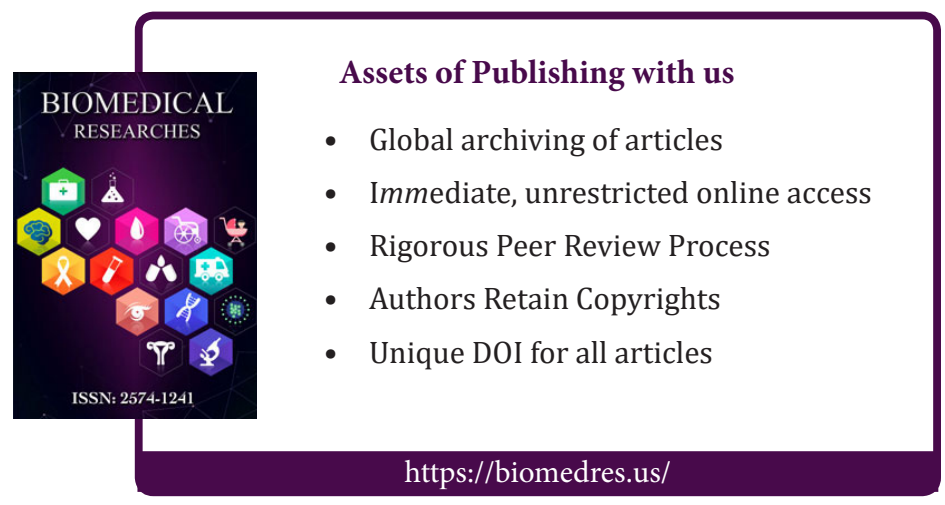

